\title{
Specific vagus nerve stimulation parameters alter serum cytokine levels in the absence of inflammation
}

Téa Tsaava ${ }^{1 \dagger}$, Timir Datta-Chaudhuri ${ }^{2,3,4 \dagger}$, Meghan E. Addorisio ${ }^{1}$, Emily Battinelli Masi ${ }^{1,3}$, Harold A. Silverman ${ }^{1}$, Justin E. Newman ${ }^{1}$, Gavin H. Imperato ${ }^{1,4}$, Chad Bouton ${ }^{2,3,4}$, Kevin J. Tracey ${ }^{1,2,3,4^{*}}$, Sangeeta S. Chavan ${ }^{1,2,3,4^{*}}$ and Eric H. Chang ${ }^{1,2,3^{*}}$

\begin{abstract}
Background: Electrical stimulation of peripheral nerves is a widely used technique to treat a variety of conditions including chronic pain, motor impairment, headaches, and epilepsy. Nerve stimulation to achieve efficacious symptomatic relief depends on the proper selection of electrical stimulation parameters to recruit the appropriate fibers within a nerve. Recently, electrical stimulation of the vagus nerve has shown promise for controlling inflammation and clinical trials have demonstrated efficacy for the treatment of inflammatory disorders. This application of vagus nerve stimulation activates the inflammatory reflex, reducing levels of inflammatory cytokines during inflammation.

Methods: Here, we wanted to test whether altering the parameters of electrical vagus nerve stimulation would change circulating cytokine levels of normal healthy animals in the absence of increased inflammation. To examine this, we systematically tested a set of electrical stimulation parameters and measured serum cytokine levels in healthy mice.

Results: Surprisingly, we found that specific combinations of pulse width, pulse amplitude, and frequency produced significant increases of the pro-inflammatory cytokine tumor necrosis factor (TNF), while other parameters selectively lowered serum TNF levels, as compared to sham-stimulated mice. In addition, serum levels of the antiinflammatory cytokine interleukin-10 (IL-10) were significantly increased by select parameters of electrical stimulation but remained unchanged with others.

Conclusions: These results indicate that electrical stimulation parameter selection is critically important for the modulation of cytokines via the cervical vagus nerve and that specific cytokines can be increased by electrical stimulation in the absence of inflammation. As the next generation of bioelectronic therapies and devices are developed to capitalize on the neural regulation of inflammation, the selection of nerve stimulation parameters will be a critically important variable for achieving cytokine-specific changes.
\end{abstract}

Keywords: Inflammatory reflex, Neuromodulation, Tumor necrosis factor, Interleukin-10

\footnotetext{
*Correspondence: kjtracey@northwell.edu; schavan@northwell.edi; echang1@northwell.edu

†Téa Tsaava and Timir Datta-Chaudhuri are co-first authors.

${ }^{1}$ Laboratory of Biomedical Science, Feinstein Institutes for Medical Research, Northwell Health, 350 Community Drive, Manhasset, NY 11030, USA

Full list of author information is available at the end of the article
}






\section{Background}

Electrical stimulation is an important neuromodulation technique used to activate nerves and muscles within the body. When electrical current is applied to nerve tissue using electrodes, there are multiple variables that determine whether the desired neural fibers are activated. In general, fiber activation by electrical stimulation follows a recruitment order from largest to smallest, with the smallest diameter fibers requiring the highest stimulation current levels (Baratta et al., 1989; Blair et al., 1933; Fang and Mortimer, 1991). Because diverse fiber types in the peripheral nervous system innervate different target organs and exhibit distinct functions, this fiber recruitment principle is important to achieve the desired physiological changes from activating nerves (Gorman and Mortimer, 1983). Electrical stimulation parameters such as output frequency, current, duration, and amplitude, are important determinants for achieving selective and efficient nerve activation (Grill, 2015).

In the vagus nerve, there are three main fiber types: A-, B-, and C-fibers that can be distinguished on the basis of axon diameters, myelination, conduction velocity and stimulation thresholds for activation (Heck et al., 2002; Groves and Brown, 2005). This mixed nerve carries sensory afferent and motor efferent signals between the brain and the body to mediate vital functions of the autonomic nervous system (Bonaz et al., 2013; Tracey, 2002). A large body of preclinical studies and emerging clinical evidence indicates that electrical stimulation at the cervical vagus nerve is able to change the body's immune response to injury or infection, specifically by reducing the levels of certain serum cytokines that are important mediators of inflammation in the body (Andersson and Tracey, 2012; Borovikova et al., 2000). This stimulation of the vagus nerve regulates cytokine release from the spleen through activation of the inflammatory reflex, thereby protecting against lethality in models of systemic inflammation (Borovikova et al., 2000; Chavan et al., 2017; Tracey, 2002). Early clinical evidence for the therapeutic efficacy of this electrical vagus nerve stimulation has shown promise to treat patients with chronic inflammatory disorders (Bonaz et al., 2016; Koopman et al., 2016). Previous studies have also demonstrated that electrical stimulation parameters can be modified to intentionally elicit different physiological effects, such as the separation of anti-inflammatory and cardioinhibitory effects (Huston et al., 2007). The antiinflammatory effects of vagus nerve stimulation have been attributed to A- and B-fiber activation, while the cardioinhibitory effects are thought to be mediated by only B-fibers (Huston et al., 2007; Olofsson et al., 2015; Yoo et al., 2016).

As nerve stimulation parameters affect differential fiber recruitment, we reasoned that specific stimulation parameters might affect serum cytokine levels in a parameter-dependent fashion. We modified the output of our electrical stimulator to select frequencies, amplitudes, and pulse widths predicted to differentially activate different classes of fibers within the vagus nerve. Here we observed that specific combinations of frequency, pulse width, and pulse amplitudes either increase or decrease certain cytokines, such as tumor necrosis factor alpha (TNF $)$ and interleukin 10 (IL-10) in healthy mice.

\section{Methods \\ Animals}

Naïve male BALB/c mice (8 to 12 weeks old) were obtained from Charles River Laboratories (Wilmington, MA, USA) and acclimated for at least 1 week before conducting experiments. Animals were housed on a 12: $12 \mathrm{~h}$ reverse light/dark cycle at $23^{\circ} \mathrm{C}$ and relative humidity $30-70 \%$. Mice were housed with ad libitum water and chow. All experiments were performed under protocols approved by the Institutional Animal Care and Use Committee of the Feinstein Institutes for Medical Research and in strict adherence with NIH guidelines on the care and use of laboratory animals. A total of 2040 mice were used in this study.

\section{Vagus nerve isolation and electrical stimulation}

Standard chow was withheld from animals for a period of up to $3 \mathrm{~h}$ prior to stimulation of the cervical vagus nerve. All surgical procedures were conducted using aseptic technique. Vagus nerve isolation was carried out as previously described (Silverman et al., 2018). Briefly, mice were administered isoflurane anesthesia through a nose cone in the supine position (oxygen flow $1 \mathrm{~L} / \mathrm{min}$, isoflurane 1.75\%). An appropriate depth of anesthesia was assessed by toe pinch reflex. The cervical region was shaved with an electric razor and then sterilized with $70 \%$ ethanol. A midline incision was made, the salivary glands were identified and bluntly dissected to expose the left carotid bundle lateral to the sternocleidomastoid muscle. The left cervical vagus nerve was separated from the carotid sheath and placed on a $200 \mu \mathrm{m}$ diameter micro cuff sling bipolar electrode with platinum-iridium contacts (CorTec GmbH, Freiburg, Germany). Parafilm was placed over the surgical site to prevent desiccation of the nerve during stimulation. Heart rate was continuously monitored using a MouseSTAT ${ }^{\circledast}$ Heart Rate Monitor (Kent Scientific, Torrington, CT, USA).

Electrical pulses were delivered by a constant current stimulator system PlexStim 2.0 (Plexon, Dallas, TX) and individually controlled with PlexStim v2.2 software. The following stimulation parameters were used for stimulation: Four minute duration, pulse width $(50 \mu \mathrm{s}, 250 \mu \mathrm{s})$, amplitude $(50 \mu \mathrm{A}, 200 \mu \mathrm{A}$, and $750 \mu \mathrm{A})$, and frequency 
$(30 \mathrm{~Hz}, 100 \mathrm{~Hz})$. Sham operated mice underwent the same surgical procedures but without electrical stimulation. After stimulation, the skin was sutured closed and the animals were returned to their home cages for recovery.

\section{Serum collection and analysis}

Two hours following stimulation, whole blood was collected from animals by cardiac puncture following euthanasia by $\mathrm{CO}_{2}$ asphyxiation. Blood was allowed to clot in a polypropylene tube at room temperature for 30-60 min. To obtain serum, the tubes were centrifuged two times, first at $5000 \mathrm{x} \mathrm{g}$ for $10 \mathrm{~min}$, followed by $10,000 \times \mathrm{g}$ for $2 \mathrm{~min}$. The supernatant serum was collected in a clean tube and stored at $-20^{\circ} \mathrm{C}$ until further processing. Serum was analyzed on multiplex cytokine immunoassay plates (V-PLEX Panel 1 mouse kit; Meso Scale Discovery, Rockville MD) to quantify levels of IFN- $\gamma$, IL-1 $\beta$, IL-2, IL-4, IL-5, IL-6, CXCL1, IL-10, IL-12p70, and TNF- $\alpha$.

\section{Statistical analysis}

Differences in serum cytokine levels between stimulated and non-stimulated (sham) groups were analyzed by Mann-Whitney $U$ tests (Prism 8.0). In all tests, $P<0.05$ was accepted as an indication of statistical significance.

\section{Results}

Here we utilized an established methodology to surgically isolate and electrically stimulate the vagus nerve. For all experiments, stimulation was performed on experimental animals with sham-matched controls. Following vagus nerve stimulation, mice were euthanized after $2 \mathrm{~h}$, and blood was collected (Fig. 1a). During electrical stimulation, depolarization of the nerve was achieved via cathodal current application at the electrode-tissue interface and at the electrode with the lower electrical potential $(1 \mathrm{~mm}$ spacing between electrodes). We utilized biphasic waveforms, with a secondary anodic phase to balance out the net effect charge to zero (Fig. 1b). Without charge balancing, a detrimental electrical potential buildup at the electrode may occur

A.

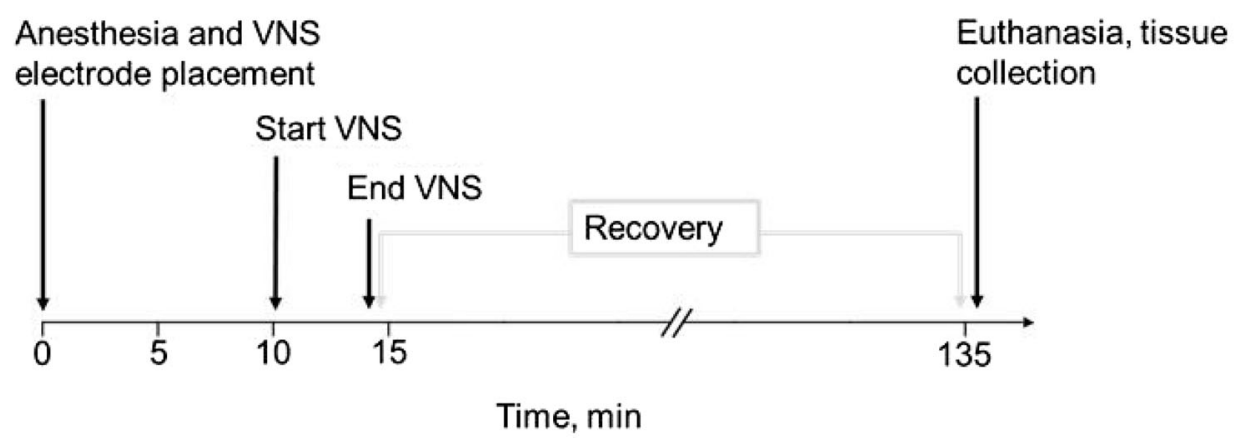

B.

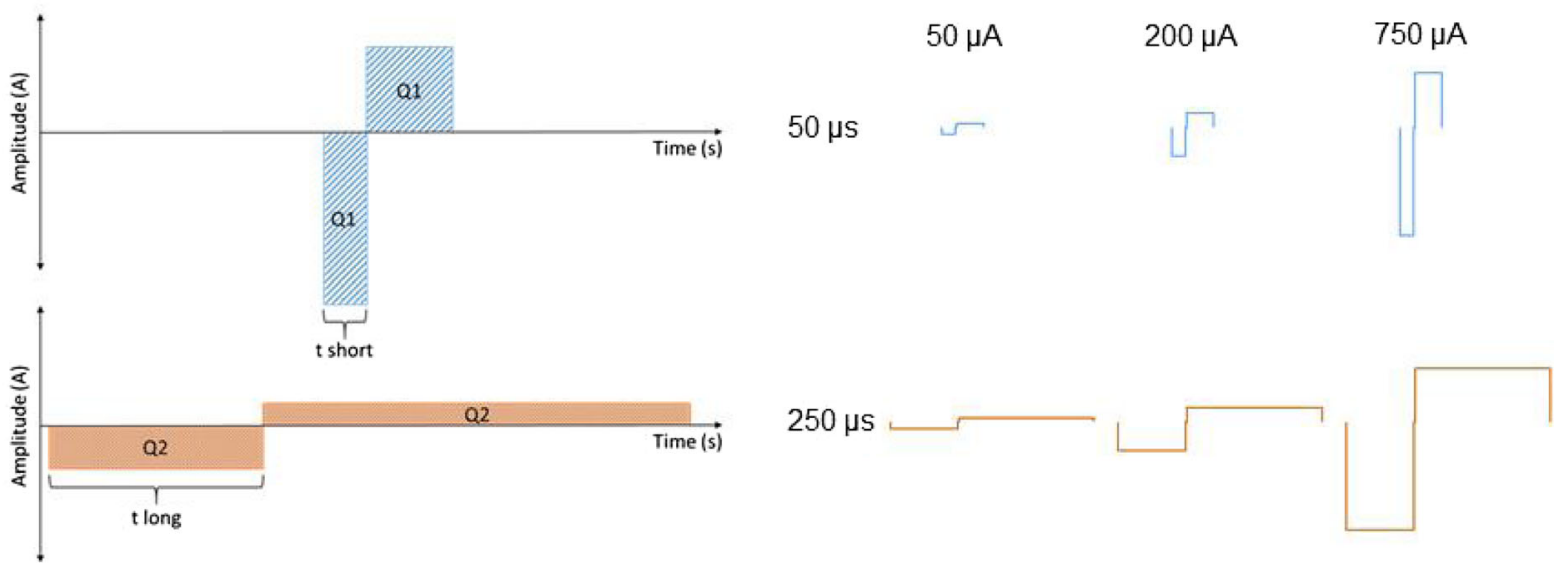

Fig. 1 Experimental design and stimulation pulse waveforms. a Experimental timeline. Electrical stimulation pulse trains were applied to the exposed left cervical vagus nerve for 4 min under anesthesia. Following stimulation, the animals were recovered for 2 h, euthanized, and whole blood was collected through cardiac puncture. $\mathbf{b}$ Schematic of the charge-balanced stimulation waveforms used during stimulation, with short pulse width (top) and long pulse width (bottom). The actual waveform shapes used in this study are shown to the right 
over time resulting in both tissue damage and reduction of electrode effectiveness. We consistently placed the electrode at the same rostral-caudal position on the vagus nerve, however, we did not monitor the cathode position on the nerve. The stimulation waveforms for the different parameters are shown in Fig. 1b.

While electrical vagus nerve stimulation is known to decrease serum TNF in a setting of increased inflammation, its effect during normal physiology is unclear. To assess this, we delivered cervical vagus nerve stimulation to normal mice and measured serum cytokines $2 \mathrm{~h}$ later. We found that specific combinations of stimulation parameters significantly changed levels of serum TNF. Specifically, stimulation at the short pulse width $(50 \mu \mathrm{s})$ at $30 \mathrm{~Hz}$ pulse and $200 \mu \mathrm{A}$ amplitude produced a significant decrease in TNF (Mann Whitney $U=216, P<0.05$; Fig. 2a). Stimulation with a $50 \mu$ s pulse width at $100 \mathrm{~Hz}$

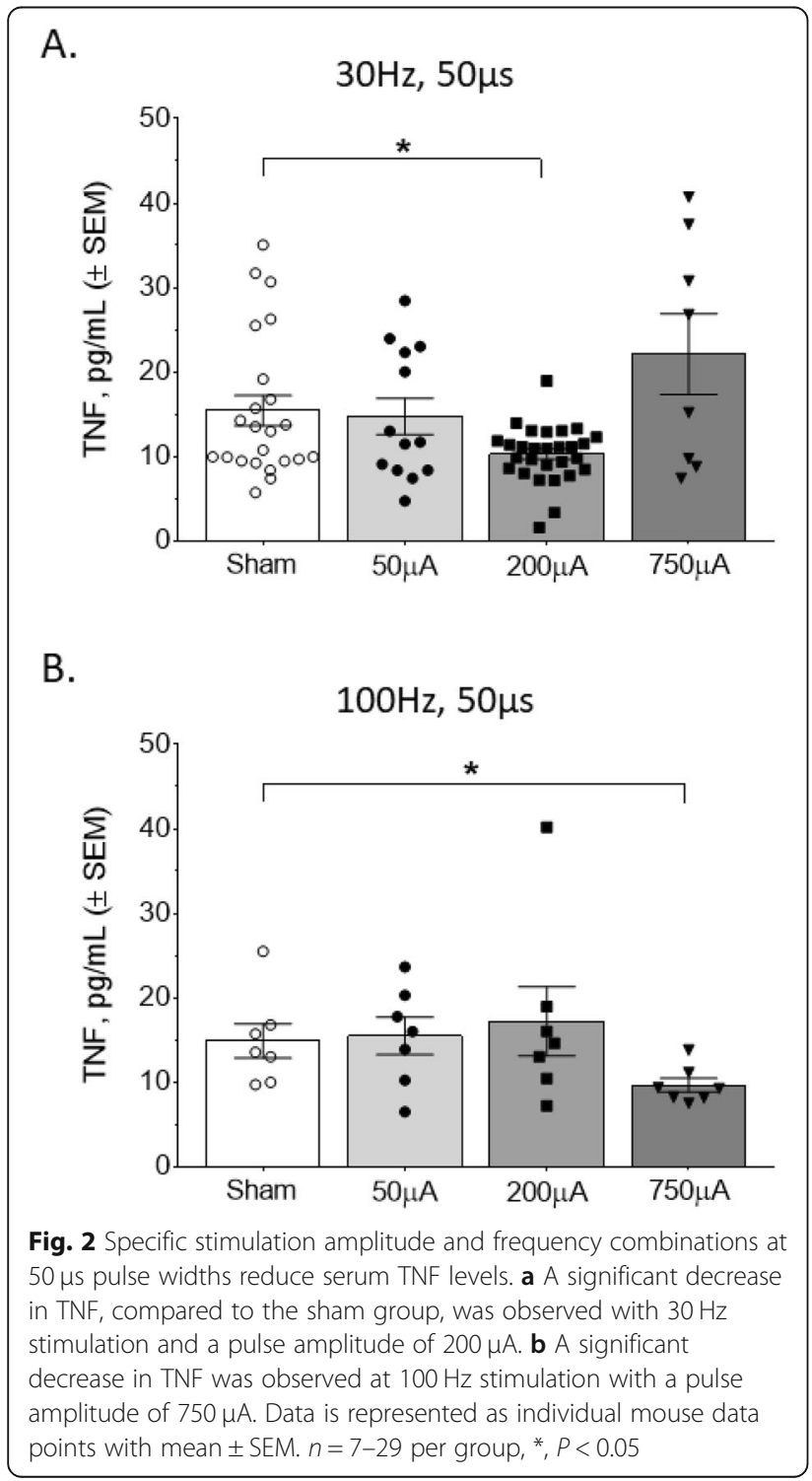

and $750 \mu \mathrm{A}$ also resulted in a decrease in serum TNF $\alpha$ (Mann Whitney $U=6, P<0.05$; Fig. 2b). When we increased the pulse width to $250 \mu$ s during stimulation, we observed a significant increase in serum TNF levels at $30 \mathrm{~Hz}$ and $750 \mu \mathrm{A}$, compared to sham mice (Mann Whitney test, $U=60, P<0.0001$; Fig. 3a). In contrast, stimulation $250 \mu$ s and $100 \mathrm{~Hz}$ produced no statistically significant changes in serum TNF (Mann Whitney $\mathrm{U}=$ $38, P=0.17$; Fig. $3 \mathrm{~b}$ ). These results suggest that specific stimulation parameters can alter serum TNF in a bidirectional manner.

IL-10 is an anti-inflammatory cytokine that suppresses Th1 cells, NK cells, and macrophages during infection (Couper et al., 2008; Hutchins et al., 2013). To examine whether electrical vagus nerve stimulation could also change levels of serum IL-10, we used specific combinations of frequency, pulse width, and amplitude followed

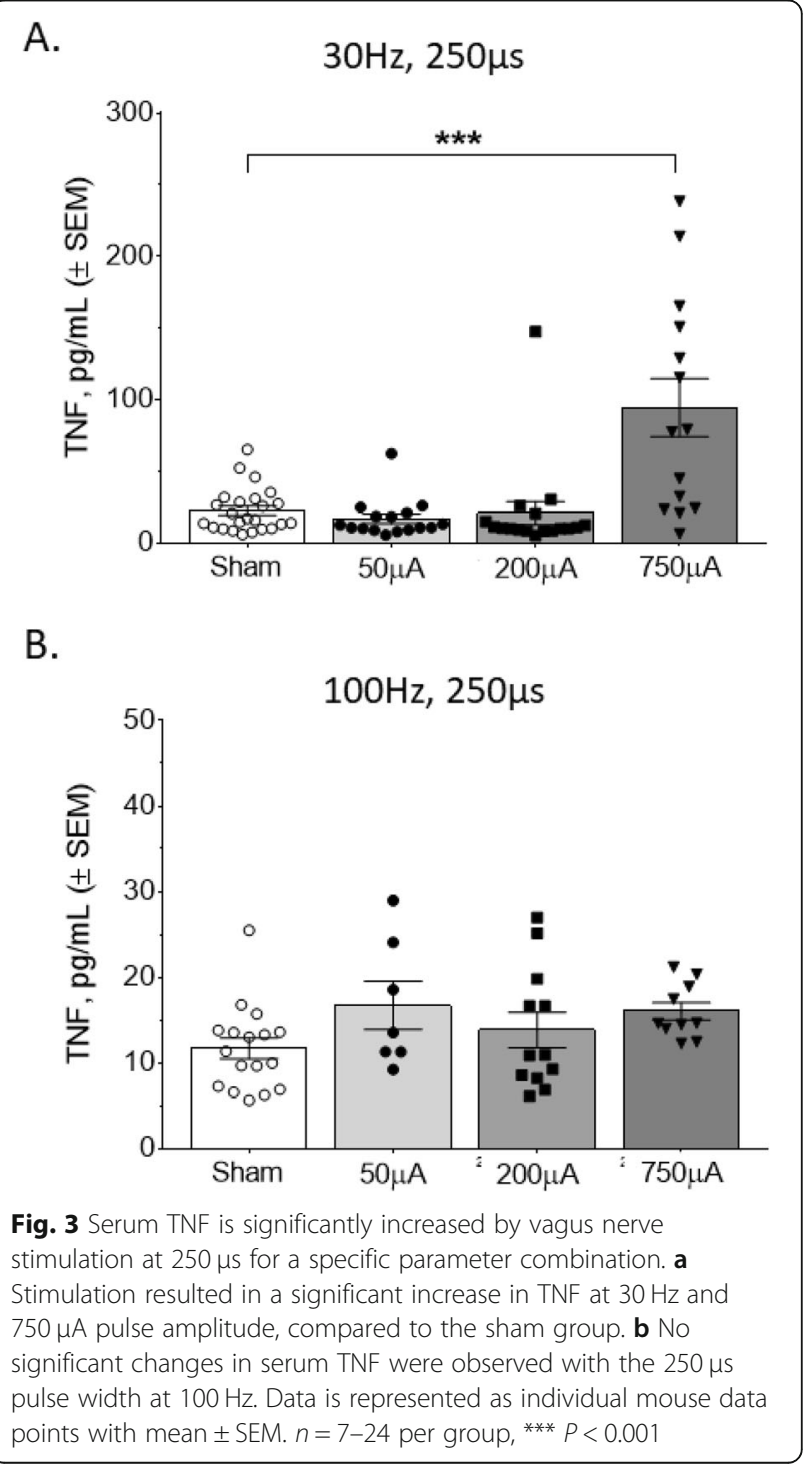


by serum IL-10 measurements. Interestingly, the shorter $50 \mu \mathrm{s}$ pulse width at $30 \mathrm{~Hz}$ produced statistically significant increases in serum IL-10 at both the $50 \mu \mathrm{A}$ and $750 \mu \mathrm{A}$ amplitudes $(50 \mu \mathrm{A}$, Mann Whitney $\mathrm{U}=115, P<$ 0.05; $750 \mu \mathrm{A}$, Mann Whitney $\mathrm{U}=53, P<0.05$; Fig. 4a). Meanwhile, a $50 \mu$ s pulse width at $100 \mathrm{~Hz}$ stimulation resulted in no significant changes in IL-10 levels (Mann Whitney $\mathrm{U}=34, P=0.96$; Fig. $4 \mathrm{~b}$ ). With the longer $250 \mu$ s pulse width, we observed statistically significant increases in IL-10 at $750 \mu \mathrm{A}$ amplitude for both the 30 $\mathrm{Hz}$ (Mann Whitney $\mathrm{U}=53, P<0.05$; Fig. $5 \mathrm{a}$ ) and $100 \mathrm{~Hz}$ frequencies (Mann Whitney $U=18, P<0.001$; Fig. $5 b$ ). There was also a significant increase in IL-10 at $100 \mathrm{~Hz}$ with the $250 \mu$ s pulse width at $50 \mu \mathrm{A}$ amplitude (Mann Whitney $U=20, P<0.05$; Fig. $5 b)$. This demonstrates that specific parameters have a different effect on serum IL-10, compared to TNF $\alpha$.

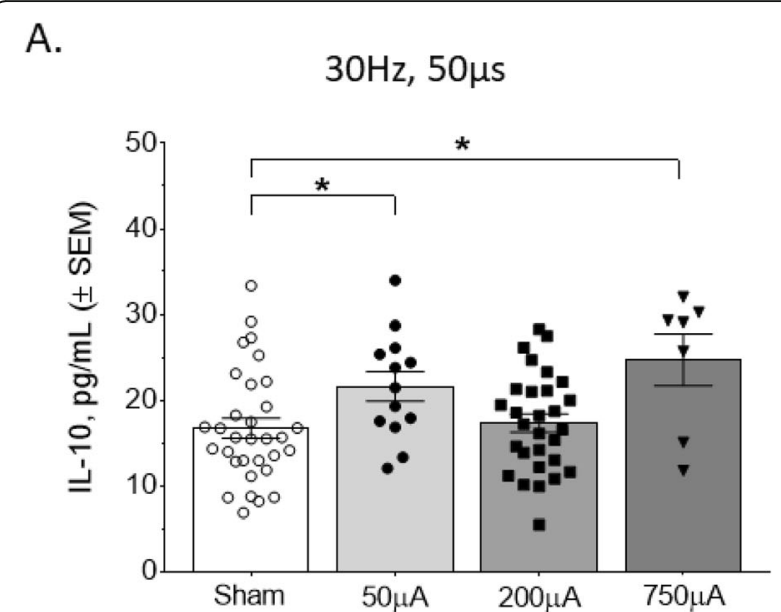

B.

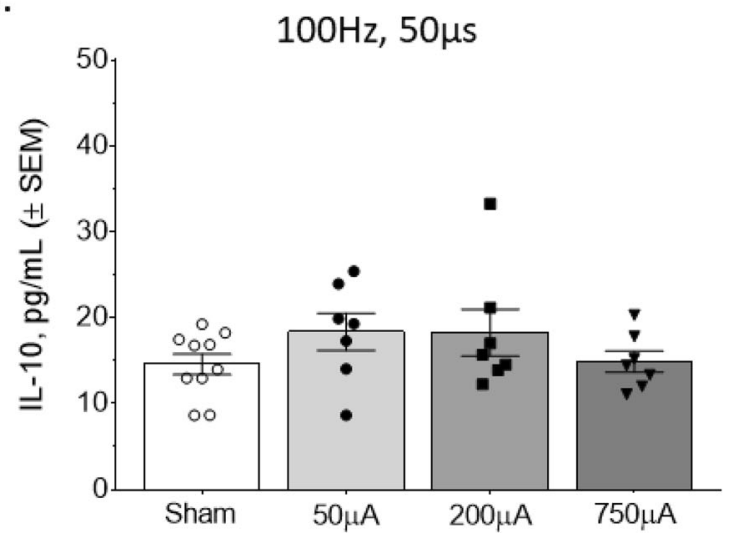

Fig. 4 Serum IL-10 is increased by select parameters of electrical stimulation with $50 \mu$ s pulse width. a Stimulation for $50 \mu \mathrm{s}$ at $30 \mathrm{~Hz}$ produced significant increases, compared to the sham group, in IL10 for both $50 \mu \mathrm{A}$ and $750 \mu \mathrm{A}$ pulse amplitudes. b No changes in serum IL-10 were observed for $100 \mathrm{~Hz}$ stimulation across the four pulse amplitudes. Data is represented as individual mouse data points with mean \pm SEM. $n=7-33$ per group, ${ }^{*}, P<0.05$

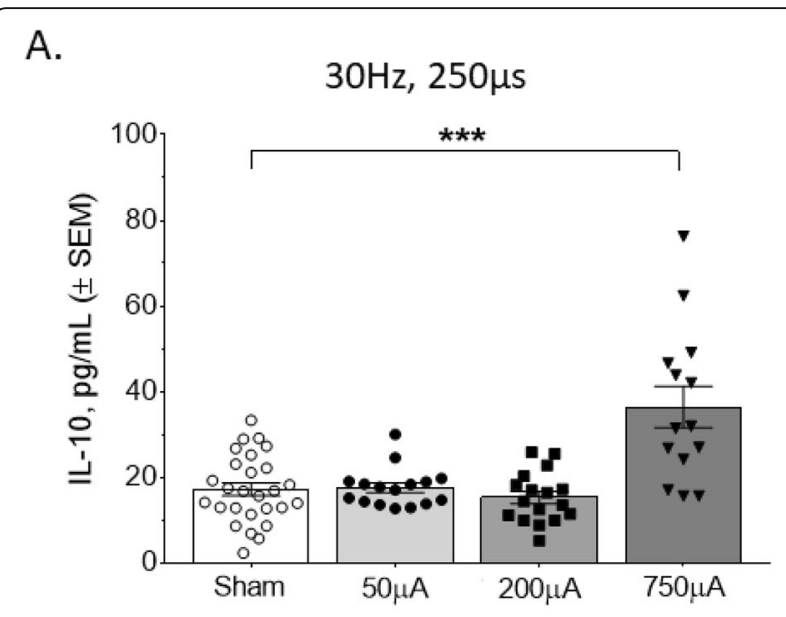

B.

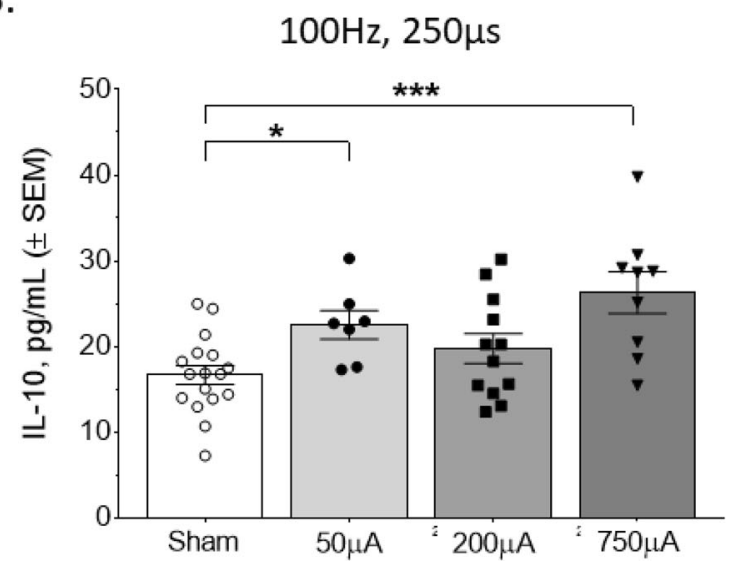

Fig. 5 Nerve stimulation with $250 \mu$ s pulse width increased serum IL-10 at several different parameters. a Stimulation with $250 \mu \mathrm{s}$ pulses at $30 \mathrm{~Hz}$ produced a marked increase in IL-10 at the $750 \mu \mathrm{A}$ pulse amplitude. b Stimulation with $250 \mu$ s pulses at $100 \mathrm{~Hz}$ produced significant increases at both $50 \mu \mathrm{A}$ and $750 \mu \mathrm{A}$ pulse amplitudes. Data is represented as individual mouse data points with mean \pm SEM. $n=7-27$ per group, ${ }^{*}, P<0.05$; ${ }^{* *} P<0.001$

Electrical nerve stimulation-induced bradycardia has been used to index nerve fiber activation during stimulation, specifically activation of the intermediate diameter B-fibers within the vagus nerve (Musselman et al., 2019; Yoo et al., 2013; Yoo et al., 2016). During our stimulation experiments, we measured heart rate with a continuous heart rate monitor to obtain an indirect measure of B-fiber recruitment. Stimulation with $50 \mu$ s pulses resulted in bradycardia only at the $750 \mu \mathrm{A}$ and $30 \mathrm{~Hz}$ parameter $(-17.1 \pm 2.9 \%$ decrease; Fig. $6 \mathrm{a})$. The $50 \mu$ s pulse width stimulation did not produce bradycardia at $100 \mathrm{~Hz}$ for any of the tested pulse widths (Fig. 6b). With the longer $250 \mu$ s pulse at $30 \mathrm{~Hz}$, bradycardia was produced at both $200 \mu \mathrm{A}$ and $750 \mu \mathrm{A}$ pulse amplitudes (Fig. 6c). At $100 \mathrm{~Hz}$, stimulation with $250 \mu$ s pulse width produced the largest measured decrease in heart rate $(-20.4 \pm$ 
A.

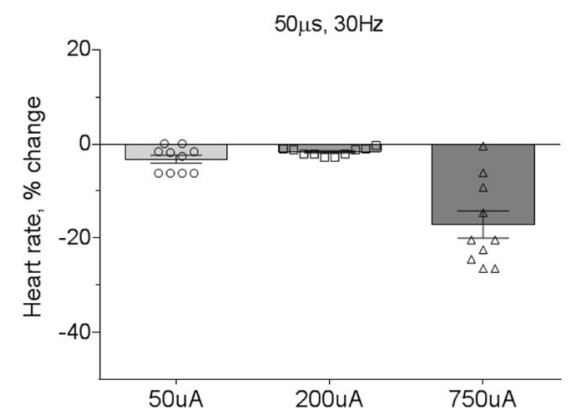

C.

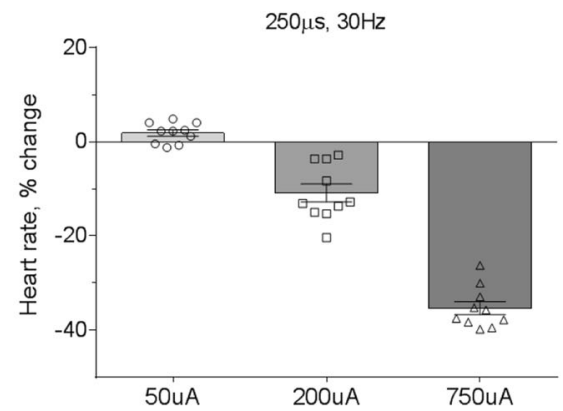

B.

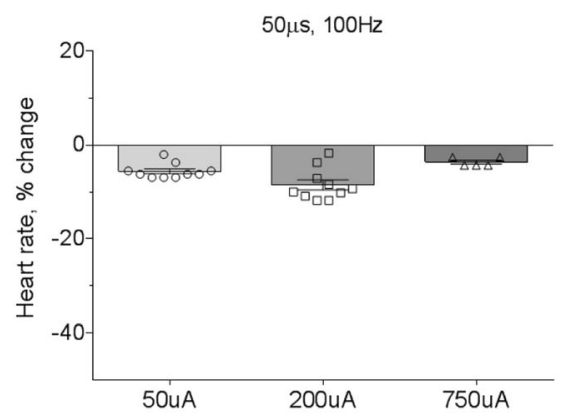

D.

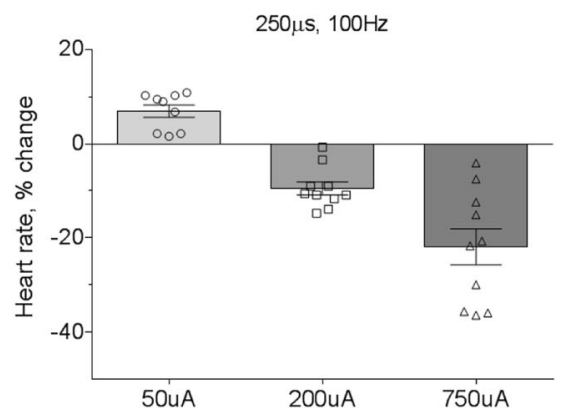

Fig. 6 The effect of different vagus nerve stimulation parameters on heart rate. a Stimulation at $30 \mathrm{~Hz}$ with $50 \mu \mathrm{s}$ pulse width resulted in bradycardia ( $\geq 10 \%$ reduction in heart rate) at only the $750 \mu \mathrm{A}$ pulse amplitude. b At $100 \mathrm{~Hz}$, bradycardia was not observed with the $50 \mu \mathrm{s}$ pulse width. c Stimulation with the longer $250 \mu$ s pulse width resulted in bradycardia at 200 and $750 \mu A$ pulse amplitudes. $\mathbf{d}$ The largest decrease in heart rate was observed with the $250 \mu$ s pulse width at $100 \mathrm{~Hz}$ and $750 \mu \mathrm{A}$ amplitude. Data is represented as individual mouse data points with mean \pm SEM. $n=5-10$ per group

4.4\%; Fig. 6d). These results indicate that alteration of stimulation parameters results in differential effects on heart rate, with longer pulse width increasing the likelihood of recruiting cardiac innervating B-fibers in the mouse vagus nerve.

In addition to assessing changes in the prototypical pro-inflammatory cytokine TNF and anti-inflammatory cytokine IL-10, we also measured serum levels of additional cytokines including: interferon gamma (IFN- $\gamma$ ), interleukin-12 p70 (IL-12 p70), interleukin-1 beta (IL1ß), interleukin-2 (IL-2), interleukin-4, (IL-4), interleukin-5 (IL-5), interleukin-6 (IL-6), and chemokine $\mathrm{C}-\mathrm{X}-\mathrm{C}$ motif ligand 1 (CXCL1). Serum levels of proinflammatory cytokines IL-6 were increased across a wide range of stimulation parameters for both the short $50 \mu$ s and long $250 \mu$ s pulse widths (Table 1, Table 2). Serum CXCL1 was also significantly increased by stimulation across several parameter combinations. For anti-inflammatory cytokines, we did not observe changes in IL-4 for any stimulation parameter combination. These results indicate that electrical stimulation may exert differential effects on the modulation of cytokines. It should be noted that the measured concentrations of IFN $\gamma$, IL-1 $\beta$, IL-2, IL-4, and IL-5 were very low and close to the sensitivity limits for the ELISA kit. Although there may be statistically significant changes with some of the stimulation parameters for these cytokines, these low levels may lack physiological relevance (Table 1, Table 2).

\section{Discussion}

The neural control of immunity through stimulation of the vagus nerve holds significant promise for treating inflammatory disorders. Here we have shown that a set of electrical stimulation parameters can change specific cytokine levels in the absence of inflammation. To our surprise, we found electrically stimulating the vagus nerve with specific parameters results in increased circulating levels of TNF and IL-10, cytokines known to be involved in inflammatory responses. These results indicate that systemic cytokines respond to specific combinations of frequency, amplitude, and pulse width applied to the cervical vagus nerve. How these parameters affect the recruitment of specific fiber types within the vagus nerve remains a topic of important ongoing work. Our results indicate that the refinement of these parameters may be important in the neuromodulation of immunological responses mediated through vagal signaling.

The effect of vagus nerve stimulation on cytokines in the absence of systemic inflammation has not, to our 
Table 1 Cytokines (mean \pm SEM) at $50 \mu$ s pulse

\begin{tabular}{|c|c|c|c|c|c|c|c|}
\hline Cytokines & Sham & $\begin{array}{l}50 \mathrm{uA} \\
30 \mathrm{~Hz}\end{array}$ & $\begin{array}{c}200 \mathrm{uA} \\
30 \mathrm{~Hz}\end{array}$ & $\begin{array}{c}750 \mathrm{uA} \\
30 \mathrm{~Hz}\end{array}$ & $\begin{array}{c}50 \mathrm{uA} \\
100 \mathrm{~Hz}\end{array}$ & $\begin{array}{l}200 \mathrm{uA} \\
100 \mathrm{~Hz}\end{array}$ & $\begin{array}{l}750 \mathrm{uA} \\
100 \mathrm{~Hz}\end{array}$ \\
\hline IFN- $\boldsymbol{Y}$ & $0.64 \pm 0.15$ & $0.48 \pm 0.13$ & $0.34 \pm 0.05$ & $0.46 \pm 0.26$ & $0.39 \pm 0.11$ & $0.53 \pm 0.21$ & $0.20 \pm 0.02$ \\
\hline IL-12 p70 & $37.27 \pm 5.72$ & $34.18 \pm 7.17$ & $47.35 \pm 6.77$ & $30.35 \pm 9.37$ & $41.24 \pm 11.16$ & $58.15 \pm 16.82$ & $43.77 \pm 10.46$ \\
\hline $\mathrm{IL}-1 \beta$ & $1.79 \pm 0.32$ & $2.43 \pm 0.41 *$ & $1.53 \pm 0.21$ & $2.03 \pm 0.38$ & $2.93 \pm 0.54$ & $2.15 \pm 0.52$ & $2.35 \pm 0.73$ \\
\hline IL-2 & $1.75 \pm 0.70$ & $1.29 \pm 0.65$ & $1.82 \pm 0.71$ & $1.40 \pm 0.35$ & $2.25 \pm 0.64$ & $2.09 \pm 0.88$ & $0.36 \pm 0.10$ \\
\hline IL-4 & $0.22 \pm 0.08$ & $0.37 \pm 0.16$ & $0.81 \pm 0.41$ & $0.47 \pm 0.28$ & $0.45 \pm 0.18$ & $0.43 \pm 0.10$ & $0.36 \pm 020$ \\
\hline IL-5 & $3.12 \pm 0.34$ & $4.58 \pm 0.89$ & $4.02 \pm 0.75$ & $4.43 \pm 0.88$ & $5.38 \pm 1.05 *$ & $4.42 \pm 0.95$ & $1.85 \pm 0.20 *$ \\
\hline IL-6 & $\begin{array}{c}2289.58 \pm \\
331.97\end{array}$ & $\begin{array}{l}3270.73 \pm \\
323.83 * *\end{array}$ & $\begin{array}{l}2653.11 \pm \\
184.78 *\end{array}$ & $\begin{array}{c}5516.24 \pm \\
1139.26^{* *}\end{array}$ & $\begin{array}{c}2826.10 \pm \\
305.77\end{array}$ & $\begin{array}{l}2921.93 \pm \\
322.54 *\end{array}$ & $\begin{array}{c}2906.27 \pm \\
320.22 *\end{array}$ \\
\hline IL-10 & $16.21 \pm 0.93$ & $21.61 \pm 1.73 *$ & $17.32 \pm 1.06$ & $24.74 \pm 3.03 *$ & $18.36 \pm 2.18$ & $18.26 \pm 2.72$ & $14.88 \pm 1.27$ \\
\hline TNF & $15.32 \pm 1.43$ & $14.76 \pm 2.14$ & $10.26 \pm 0.63^{*}$ & $22.13 \pm 4.78$ & $15.47 \pm 2.22$ & $17.19 \pm 4.09$ & $9.61 \pm 0.82^{*}$ \\
\hline CXCL1 & $739.68 \pm 50.39$ & $\begin{array}{c}1004.54 \pm \\
86.69 *\end{array}$ & $\begin{array}{l}1074.40 \pm \\
60.44 * * *\end{array}$ & $\begin{array}{c}786.88 \pm \\
71.59\end{array}$ & $\begin{array}{l}1131.30 \pm \\
71.65 * * *\end{array}$ & $\begin{array}{c}1025.21 \pm \\
68.99 * *\end{array}$ & $\begin{array}{c}880.59 \pm \\
92.48\end{array}$ \\
\hline
\end{tabular}

Full multiplex panel results of serum cytokine levels following nerve stimulation with $50 \mu$ s pulse width. Values in orange indicate significant increases while values in blue indicate significant decreases, compared to the Sham group. $n=7-29$ per group; ${ }^{*}, P<0.05 ;{ }^{* *}, P<0.01 ;{ }^{* * *} P<0.001$

knowledge, been carefully examined. This is the first demonstration that specific stimulation parameters can be used to increase serum TNF and IL-10 levels. TNF is an inflammatory cytokine that is released by macrophages following infection and injury. Transient elevations in serum TNF are required to coordinate host defense against pathogens and the repair of injured host tissue. While physiological inflammation is locally protective, pathological inflammation caused by persistent and unregulated TNF levels cause chronic tissue damage and drive the pathogenesis of inflammatory disease including rheumatoid arthritis, Crohn's disease, and inflammatory bowel disease (Bonaz et al., 2016; Kalliolias 2016; Koopman et al., 2016). A large body of work established the critical role of TNF in the pathogenesis of systemic inflammation in animal models and led to the clinical translation of TNF blockade to treat chronic inflammation. The beneficial effect of vagus nerve stimulation for reducing TNF in the context of inflammatory disorders is clear, however, an increase in proinflammatory cytokine levels may also be beneficial in certain physiological and pathologic contexts. An increase in circulating cytokines might be useful to bolster the immune system in conditions of immunosuppression, for example, due to immunodeficiencies or viral infection (Breen, 2002; Varzaneh et al., 2014). Cancer patients with lower levels of circulating cytokines may also benefit from selective neuromodulation techniques to increase those levels (Khan et al., 2019). As techniques are developed to better understand the relationship between vagus nerve activity and cytokine signaling (Steinberg et al., 2016; Tsaava et al., 2019; Zanos et al., 2018), there may also be other physiological conditions that would benefit from intentionally increasing cytokine levels.

Our results suggest that serum cytokines may likewise be controlled by specific fiber sets and firing patterns. Altering the electrical stimulation parameters affects the recruitment of specific vagus nerve fibers. A large body of evidence shows that increasing pulse width and amplitude, and consequently the charge delivered during both cathodic and anodic phases of the pulse, inhibits activation of large diameter fibers while selectively 
Table 2 Cytokines (mean \pm SEM) at $250 \mu$ s pulse

\begin{tabular}{|c|c|c|c|c|c|c|c|}
\hline Cytokines & Sham & $\begin{array}{l}50 \mathrm{uA} \\
30 \mathrm{~Hz}\end{array}$ & $\begin{array}{c}200 \mathrm{uA} \\
30 \mathrm{~Hz}\end{array}$ & $\begin{array}{c}750 \mathrm{uA} \\
30 \mathrm{~Hz}\end{array}$ & $\begin{array}{c}50 \mathrm{uA} \\
100 \mathrm{~Hz}\end{array}$ & $\begin{array}{l}200 \mathrm{uA} \\
100 \mathrm{~Hz}\end{array}$ & $\begin{array}{l}750 \mathrm{uA} \\
100 \mathrm{~Hz}\end{array}$ \\
\hline IFN-Y & $0.69 \pm 0.16$ & $0.58 \pm 0.22$ & $0.45 \pm 0.09$ & $0.96 \pm 0.48$ & $0.66 \pm 0.20$ & $0.51 \pm 0.12$ & $0.25 \pm 0.03$ \\
\hline IL-12 p70 & $50.31 \pm 15.11$ & $48.26 \pm 6.26$ & $48.83 \pm 13.70$ & $39.63 \pm 4.49$ & $52.80 \pm 13.92$ & $41.90 \pm 7.12$ & $78.17 \pm 9.33 * *$ \\
\hline IL-1 $\beta$ & $3.10 \pm 0.91$ & $5.42 \pm 2.04 * *$ & $2.01 \pm 0.45$ & $1.48 \pm 0.34$ & $4.07 \pm 0.52$ & $2.54 \pm 0.44$ & $2.15 \pm 0.29$ \\
\hline IL-2 & $2.85 \pm 1.12$ & $1.69 \pm 0.65$ & $3.05 \pm 1.23$ & $1.88 \pm 1.13$ & $5.21 \pm 2.31$ & $4.80 \pm 2.11$ & $2.63 \pm 0.56$ \\
\hline IL-4 & $0.34 \pm 0.08$ & $0.44 \pm 0.22$ & $0.38 \pm 0.17$ & $0.54 \pm 0.29$ & $0.67 \pm 0.42$ & $0.53 \pm 0.21$ & $0.20 \pm 0.06$ \\
\hline IL-5 & $2.97 \pm 0.36$ & $4.36 \pm 0.72$ & $5.48 \pm 1.26 *$ & $6.13 \pm 1.41 *$ & $9.50 \pm 2.74 * *$ & $6.19 \pm 1.20 * * *$ & $3.55 \pm 0.65$ \\
\hline IL-6 & $\begin{array}{c}2125.23 \pm \\
243.76\end{array}$ & $\begin{array}{c}2981.44 \pm \\
376.14 *\end{array}$ & $\begin{array}{c}2911.30 \pm \\
405.83 *\end{array}$ & $\begin{array}{c}5880.09 \pm \\
1248.88 * * *\end{array}$ & $\begin{array}{l}3541.48 \pm \\
408.73 * *\end{array}$ & $\begin{array}{c}3040.72 \pm \\
394.06 *\end{array}$ & $\begin{array}{c}5490.44 \pm \\
473.28 * * * *\end{array}$ \\
\hline IL-10 & $16.99 \pm 1.02$ & $17.57 \pm 1.15$ & $15.31 \pm 1.43$ & $36.44 \pm 4.82 * * *$ & $22.54 \pm 1.68 *$ & $19.75 \pm 1.73$ & $26.31 \pm 2.46^{* * *}$ \\
\hline TNF & $17.93 \pm 2.05$ & $16.74 \pm 3.39$ & $20.64 \pm 8.09$ & $\begin{array}{c}94.27 \pm \\
20.30 * * *\end{array}$ & $16.70 \pm 2.82$ & $13.83 \pm 2.06$ & $16.03 \pm 1.02$ \\
\hline CXCL1 & $\begin{array}{c}798.88 \pm \\
55.16\end{array}$ & $\begin{array}{c}947.72 \pm \\
83.90\end{array}$ & $\begin{array}{c}1134.26 \pm \\
121.55 *\end{array}$ & $\begin{array}{c}907.32 \pm \\
114.89\end{array}$ & $\begin{array}{l}1192.14 \pm \\
80.46 * * *\end{array}$ & $\begin{array}{c}1717.80 \pm \\
170.34 * * * *\end{array}$ & $\begin{array}{c}1081.02 \pm \\
160.75\end{array}$ \\
\hline
\end{tabular}

Full multiplex panel results of serum cytokine levels following nerve stimulation with $250 \mu$ s pulse width. Values in orange indicate significant increases, compared to the Sham group. $n=9-33$ per group; ${ }^{*}, P<0.05 ;{ }^{* *}, P<0.01 ;{ }^{* * *} P<0.001 ; * * *, P<0.0001$

activating smaller diameter fibers (Baratta et al., 1989; Musselman et al., 2019). For example, lower amplitude stimulation activates A-fibers but as stimulation increases, the A-fiber activation becomes suppressed and B-fibers become activated to change heart rate (Burke et al., 1975). It is possible that the differential effect on heart rate may be a result of effective recruitment of large and small fiber types with the long pulse at lower amplitudes, and selective inhibition of larger fibers at the larger pulse widths. The effective pulse widths and stimulus amplitudes necessary to elicit heart rate changes is shown in Fig. 6. As the stimulation charge increased, we generally observed more pronounced bradycardia, however, heart rate decreases were not always coupled to changes in serum cytokines. The largest increase in serum TNF at $250 \mu$ s pulse width, $30 \mathrm{~Hz}$, and $750 \mu \mathrm{A}$ was associated with the largest decrease in heart rate. However, TNF levels did not change for two other stimulation parameters that induced bradycardia (Fig. 6: $50 \mu$ s pulse width, $30 \mathrm{~Hz}, 750 \mu \mathrm{A}$ and $250 \mu$ s pulse width, $100 \mathrm{~Hz}, 750 \mu \mathrm{A})$. This dissociation between bradycardia and serum cytokines levels confirms prior work indicating that cytokine effects are independent of the B-fiber activation that induces bradycardia (Huston et al., 2007). While the specific vagus nerve fibers mediating the inflammatory reflex are not known, they are thought to be cholinergic efferents descending to peripheral ganglia (Bonaz et al., 2013; Chavan et al., 2017). These cholinergic fibers are myelinated and intermediate in diameter (i.e. A- or B-fibers), and are therefore targeted for recruitment by stimulation pulses of moderate width and amplitude (Olofsson et al., 2015).

We have considered the possibility that different durations of total stimulation may also be an important variable in cytokine-specific modulation via the vagus nerve. The frequency of stimulation also plays a role in fiber recruitment. While not examined in this study, lower frequency stimulation $(1-20 \mathrm{~Hz})$ may also alter cytokine levels. Further, while the applicability of these findings may be most relevant for disease conditions, these studies were carried out in healthy control animals. Therefore, they should be interpreted with caution if trying to 
extrapolate to specific disease conditions. The parameter space of electrical nerve stimulation is important because varying time, duration, and amplitude should affect activation of different target organs and brain structures. Electrical stimulation delivered to cervical vagus nerve is broadly applied to all nerve fibers, including sensory afferents and motor efferents. It is thought that B-fibers mediate motor efferent control of the visceral organs. Because the vagus nerve innervates several major organs in the viscera, stimulation of this neve has the potential to treat a broad range of disorders ranging including but not limited to arthritis, myocardial infarctions, and metabolic disorders (Kong et al., 2012; Levine et al., 2014; Chang et al., 2019).

\section{Conclusions}

The unexpected results here indicate that it is possible to stimulate the production of TNF and other cytokines using electrical stimulation of the cervical vagus nerve in normal, non-inflammatory conditions. As bioelectronic therapies continue to evolve for controlling inflammation, increasingly refined stimulation strategies will be needed to activate fibers of interest (Grill, 2015; Tsaava et al., 2019), while also minimizing the potential for the negative outcomes and off-target effects associated with vagus nerve stimulation. The effectiveness of these selective stimulation paradigms will depend on knowing which specific fiber populations mediate the neural regulation of inflammation and the optimal techniques for activating them. Our results show that varying stimulation parameters of frequency, amplitude, and pulse width holds promise for the specific regulation of cytokine responses in both health and disease.

\section{Abbreviations}

CXCL1: Chemokine ligand C-X-C motif ligand 1; IFNץ: Interferon gamma; IL10: Interleukin-10; IL-12 p70: Interleukin-12 p70; IL-13: Interleukin-1 beta; IL2: Interleukin-2; IL-4: Interleukin-4; IL-5: Interleukin-5; IL-6: Interleukin-6;

TNFa: Tumor necrosis factor alpha

\section{Acknowledgements}

Not applicable.

\section{Authors' contributions}

T.T., M.E.A., E.B.M., H.A.S., J.E.N., and E.H.C. performed experiments and analyzed data. T.T., C.B., S.S.C, and K.J.T. designed the experiments. T.T., T.D., G.H.I., K.J.T., S.S.C., and E.H.C. wrote and finalized the manuscript. The author(s) read and approved the final manuscript.

\section{Funding}

This work was supported in part by DARPA (HR0011-15-2-0016) and NIH (1R35GM118182-01).

\section{Availability of data and materials \\ Datasets used in this study are available from the corresponding author upon reasonable request.}

\section{Ethics approval and consent to participate}

This study was conducted with approval from the Feinstein Institutes IACUC and in accordance with $\mathrm{NIH}$ guidelines for the care and use of laboratory animals.
Consent for publication

All authors have consented to publication.

\section{Competing interests}

K.J.T. and S.S.C. hold patents broadly related to this work. They have assigned all rights to the Feinstein Institutes for Medical Research.

\section{Author details}

${ }^{1}$ Laboratory of Biomedical Science, Feinstein Institutes for Medical Research, Northwell Health, 350 Community Drive, Manhasset, NY 11030, USA.

${ }^{2}$ Institute of Bioelectronic Medicine, Feinstein Institutes for Medical Research, Northwell Health, 350 Community Drive, Manhasset, NY 11030, USA. ${ }^{3}$ Donald and Barbara Zucker School of Medicine at Hofstra/Northwell, 500 Hofstra University, Hempstead, New York 11030, USA. ${ }^{4}$ The Elmezzi Graduate School of Molecular Medicine, 350 Community Drive, Manhasset, NY 11030, USA.

Received: 15 January 2020 Accepted: 3 March 2020

Published online: 10 April 2020

\section{References}

Andersson U, Tracey KJ. Neural reflexes in inflammation and immunity. J Exp Med. 2012:209:1057-68.

Baratta R, Ichie I, Hwang SK, Solomonow M. Orderly stimulation of skeletal muscle motor units with tripolar nerve cuff electrode. IEEE Trans Biomed Eng. 1989;36(8):836-43.

Blair EA, Erlanger J. A comparison of the characteristics of axons through their individual electrical responses. Am J Physiology-Legacy Content. 1933;106(3): $524-64$.

Bonaz B, Picq C, Sinniger V, Mayol JF, Clarencon D. Vagus nerve stimulation: from epilepsy to the cholinergic anti-inflammatory pathway. Neurogastroenterol Motil. 2013;25(3):208-21. https://doi.org/10.1111/nmo.12076.

Bonaz B, Sinniger V, Hoffmann D, Clarençon D, Mathieu N, Dantzer C, Vercueil L, Picq C, Trocmé C, Faure P, Cracowski J-L, Pellissier S. Chronic vagus nerve stimulation in Crohn's disease: a 6-month follow-up pilot study. Neurogastroenterol Motil. 2016;28(6):948-53. https://doi.org/10.1111/nmo. 12792

Borovikova LV, Ivanova S, Zhang M, Yang H, Botchkina Gl, Watkins LR, Wang $H$, Abumrad N, Eaton JW, Tracey KJ. Vagus nerve stimulation attenuates the systemic inflammatory response to endotoxin. Nature. 2000;405(6785):45862.

Breen EC. Pro- and anti-inflammatory cytokines in human immunodeficiency virus infection and acquired immunodeficiency syndrome. Pharmacol Ther. 2002;95(3):295-304.

Burke D, Mackenzie RA, Skuse NF, Lethlean AK. Cutaneous afferent activity in median and radial nerve fascicles: a microelectrode study. J Neurol Neurosurg Psychiatry. 1975:38(9):855-64.

Chang EH, Chavan SS, Pavlov VA. Cholinergic control of inflammation, metabolic dysfunction, and cognitive impairment in obesity-associated disorders: mechanisms and novel therapeutic opportunities. Front Neurosci. 2019;13: 263.

Chavan SS, Pavlov VA, Tracey KJ. Mechanisms and therapeutic relevance of Neuro-immune communication. Immunity. 2017;46:927-42.

Couper KN, Blount DG, Riley EM. IL-10: the master regulator of immunity to infection. J Immunol. 2008;180:5771-7.

Fang ZP, Mortimer JT. Selective activation of small motor axons by quasitrapezoidal current pulses. IEEE Trans Biomed Eng. 1991;38(2):168-74.

Gorman PH, Mortimer JT. The effect of stimulus parameters on the recruitment characteristics of direct nerve stimulation. IEEE Trans Biomed Eng. 1983;30(7): $407-14$.

Grill WM. Model-based analyses and design of waveforms for efficient neural stimulation. Prog Brain Res. 2015;222:147-62. https://doi.org/10.1016/bs.pbr. 2015.07.031.

Groves DA, Brown VJ. Vagal nerve stimulation: a review of its applications and potential mechanisms that mediate its clinical effects. Neurosci Biobehav Rev. 2005;29:493-500. https://doi.org/10.1016/j.neubiorev.2005.01.004.

Heck C, Helmers SL, DeGiorgio CM. Vagus nerve stimulation therapy, epilepsy, and device parameters: scientific basis and recommendations for use. Neurology. 2002;59(6 Suppl. 4):S31-7.

Huston JM, Gallowitsch-Puerta M, Ochani M, Ochani K, Yuan R, Rosas-Ballina M, Ashok M, Goldstein RS, Chavan SS, Pavlov VA, Metz CN, Yang H, Czura CJ, Wang H, Tracey KJ. Transcutaneous vagus nerve stimulation reduces serum 
high mobility group box 1 levels and improves survival in murine sepsis. Crit Care Med. 2007:35:2762-8.

Hutchins AP, Diez D, Miranda-Saavedra D. The IL-10/STAT3-mediated anti inflammatory response: recent developments and future challenges. Brief Funct Genomics. 2013;12:489-98.

Kalliolias GD, Ivashkiv LB. TNF biology, pathogenic mechanisms and emerging therapeutic strategies. Nat Revi Rheumatol. 2016;12:49-62.

Khan S, Khan SA, Luo X, Fattah FJ, Saltarski J, Gloria-McCutchen Y, Lu R, Xie Y, Li $\mathrm{Q}$, Wakeland $\mathrm{E}$, Gerber DE. Immune dysregulation in cancer patients developing immune-related adverse events. Br J Cancer. 2019;120(1):63-8. https://doi.org/10.1038/s41416-018-0155-1.

Kong SS, Liu JJ, Hwang TC, Yu XJ, Zhao M, Zhao M, Yuan BX, Lu Y, Kang YM, Wang B, Zang WJ. Optimizing the parameters of vagus nerve stimulation by uniform design in rats with acute myocardial infarction. PLoS One. 2012; 7(11):e42799. https://doi.org/10.1371/journal.pone.0042799.

Koopman FA, Chavan SS, Miljko S, Grazio S, Sokolovic S, Schuurman PR, Mehta AD, Levine YA, Faltys M, Zitnik R, Tracey KJ, Tak PP. Vagus nerve stimulation inhibits cytokine production and attenuates disease severity in rheumatoid arthritis. Proc Natl Acad Sci U S A. 2016;113:8284-9.

Levine YA, Koopman FA, Faltys M, Caravaca A, Bendele A, Zitnik R, Vervoordeldonk MJ, Tak PP. Neurostimulation of the cholinergic antiinflammatory pathway ameliorates disease in rat collagen-induced arthritis. PLoS One. 2014;9:e104530

Musselman ED, Pelot NA, Grill WM. Empirically based guideline for selecting vagus nerve stimulation parameters in epilepsy and heart failure. Cold Spring Harb Perspect Med. 2019:9(7). https://doi.org/10.1101/cshperspect.a034264.

Olofsson PS, Levine YA, Caracava A, Chavan SS, Pavlov VA, Faltys M, Tracey KJ. Single-pulse and unidirectional electrical activation of the cervical Vagus nerve reduces tumor necrosis factor in Endotoxemia. Bioelectronic Med. 2015;2:37-42

Silverman HA, Stiegler A, Tsaava T, Newman J, Steinberg BE, Battinelli Masi E, Robbiati S, Bouton C, Huerta PT, Chavan SS, Tracey KJ. Standardization of methods to record Vagus nerve activity in mice. Bioelectron Med. 2018;4:3. https://doi.org/10.1186/s42234-018-0002-y.

Steinberg BE, Silverman HA, Robbiati S, Gunasekaran MK, Tsaava T, Battinelli E, Stiegler A, Bouton CE, Chavan SS, Tracey K, Huerta PT. Cytokine-specific neurograms in the sensory vagus nerve. Bioelectron Med. 2016;3:7-17.

Tracey KJ. The inflammatory reflex. Nature. 2002;420(6917):853-9.

Tsaava T, Kressel AM, Uryu K, Chavan SS, Tracey KJ, Chang EH. Optogenetic activation of fiber-specific compound action potentials in the mouse vagus nerve. 2019 9th International IEEE/EMBS Conference on Neural Engineering (NER); 2019. p. 867-70. https://doi.org/10.1109/ NER.2019.8717050.

Varzaneh FN, Keller B, Unger S, Aghamohammadi A, Warnatz K, Rezaei N. Cytokines in common variable immunodeficiency as signs of immune Dysregulation and potential therapeutic targets - a review of the current knowledge. J Clin Immunol. 2014;34:524-43. https://doi.org/10.1007/s10875014-0053-0.

Yoo PB, Lio H, Hincapie JG, Ruble SB, Hamann JJ, Grill WM. Modulation of heart rate by temporally patterned vagus nerve stimulation in anesthetized dog. Physiol Rep. 2016:4(2):e12689.

Yoo PB, Lubock NB, Hincapie JG, Ruble SB, Hamann JJ, Grill WM. High-resolution measurement of electrically-evoked vagus nerve activity in the anesthetized dog. J Neural Eng. 2013;10(2):026003. https://doi.org/10.1088/1741-2560/10/ 2/026003.

Zanos TP, Silverman HA, Levy T, Tsaava T, Battinelli E, Lorraine PW, Ashe JM, Chavan SS, Tracey K, Bouton CB. Identification of cytokine-specific sensory neural signals by decoding murine vagus nerve activity. Proc Ntl Acad Sci. 2018;115:E4843-52

\section{Publisher's Note}

Springer Nature remains neutral with regard to jurisdictional claims in published maps and institutional affiliations.

Ready to submit your research? Choose BMC and benefit from:

- fast, convenient online submission

- thorough peer review by experienced researchers in your field

- rapid publication on acceptance

- support for research data, including large and complex data types

- gold Open Access which fosters wider collaboration and increased citations

- maximum visibility for your research: over $100 \mathrm{M}$ website views per year

At BMC, research is always in progress.

Learn more biomedcentral.com/submissions 